Letter to the Editor

\title{
Rare cardiac tumor simulating ST elevated acute myocardial infarction
}

\author{
Bruno Marmelo a,*, Davide Moreira a, Anne Delgado a , Luís Abreu a , Emanuel Correia a , Miguel Correia a \\ Pedro Ferreira ${ }^{\mathrm{a}}$, Antonio Marinho da Silva ${ }^{\mathrm{b}}$, Jorge Oliveira Santos ${ }^{\mathrm{a}}$ \\ a Centro Hospitalar Tondela-Viseu, Serviço de Cardiologia, Portugal

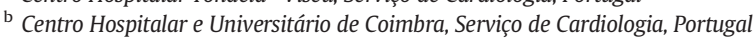

\section{A R T I C L E I N F O}

\section{Article history:}

Received 8 June 2014

Accepted 27 July 2014

Available online 4 August 2014

\section{Keywords:}

Cardiac

mass

tumor

st elevation

clear cell sarcoma

A 57-year-old Caucasian woman, appealed to our hospital with a typical pleuritic chest pain. The patient was in good condition and no abnormalities on physical examination with the exception for a sinus tachycardia (110/min) were noticed. Electrocardiogram which was subsequently performed revealed a convex ST segment elevation in leads V1-V5 (up to $3 \mathrm{~mm}$ ) suggesting acute myocardial infarction (Fig. 1). However, blood tests showed negative troponin I and myoglobin. CRP was elevated to $21.3 \mathrm{mg} / \mathrm{dL}$.

Otherwise she related mild systemic hypertension and a history of foot surgery (for cutaneous lesion?) 7 years ago.

As clinical and blood tests suggested a non-ischemic etiology, this one showed a circumferential pericardial effusion being significant at the left ventricular free wall, with good global and regional LV contractility. The parasternal long axis view showed severe septal hypertrophy protruding inside the right ventricle (Fig. 2). At parasternal short axis view this hypertrophy looks like a heterogeneous mass of the septum and the anterior wall (Fig. 3). Then a suspicion for heart tumor was then considered.

The addition of contrast (Sonovue ${ }^{\circledR}$ ) enhanced the heterogenic aspect and a cleavage plane between the mass and the normal myocardium (Fig. 4).

The patient had no specific clinical organ complaints other than the thoracic pain. A targeted physical examination was performed, showing no relevant abnormalities, including lymphadenopathy. Thoracic CT angiography confirmed a heterogeneous infiltrative mass of $5.9 \times$ $4.1 \mathrm{~cm}$ located in the anterior wall, mainly involving the right ventricle

\footnotetext{
* Corresponding author at: Serviço de Cardiologia do Centro Hospitalar Tondela Viseu, Avenida Rei Dom Duarte, 3504-509 Viseu, Portugal.
}

(RV), apex and the interventricular septum (IVS). Some lung parenquimal nodes $<10 \mathrm{~mm}$ were suggestive of metastasis (Fig. 5).

An abdominopelvic CT excluded any primary tumor in those locations. All tumor markers were negative.

The patient was referenced to a cardiac surgery center where they biopsied an epicardial exophytic lesion. Also a pleuropericardic window of $4 \times 4 \mathrm{~cm}$ was open. Pleuritic pain disappeared thereafter. Histology of the biopsy material revealed only a fibrin mass and a chronic collageneous pericarditis. Since the histopathological result didn't match with the imagiological findings, a cardiac MR was performed: a voluminous mass of $9 \times 9 \times 7 \mathrm{~cm}$ involving the IVS and anterior wall of the RV, with peri- and epicardial extension, and a sarcomatous appearance (Fig. 6) was reported. Lung nodes were also related as pulmonary metastasis, the biggest measuring $23 \mathrm{~mm}$.

Given the unsuccessful surgery, the will of the patient for a less invasive diagnostic method and the infiltrative aspect of the mass, right ventricle catheterization with endomyocardial biopsy was scheduled. A $23 \mathrm{~mm} \mathrm{Hg}$ systolic gradient trough the outflow tract was registered. Ventriculography showed a small right ventricular cavity, the body and outflow tract being occupied with the mass (Fig. 7). The biopsy of 6 myocardial fragments revealed only a probable myocarditis in regression.

As the diagnosis was still obscure and the mass and the metastasis were growing fast, biopsy of the lung nodules was then carried out through lateral thoracotomy. Finally the histopathological study reported a clear cell sarcoma. With the information collected retrospectively we know now that the patient had had a primary lesion of this tumor in her left hallux, which was removed in 2006. However she was lost in follow up and never had an appointment after surgery. The information of the patient about the lesion was erroneous regarding the malignancy and the tissue affected.

Clear cell sarcomas are very rare slow-growing tumors with highgrade malignancy, being also known as soft tissue melanoma. The primary lesion of this tumor occurs generally in the extremities, mostly knee, ankle and foot. Typically late metastasis and tumor recurrence are frequent. Given the rarity of this tumor there are no randomized therapeutic trials and the response to chemotherapy is rare.

The patient died 7 months after the initial diagnosis.

\section{Conflict of interest}

The authors report no relationships that could be construed as a conflict of interest. 


\section{a}

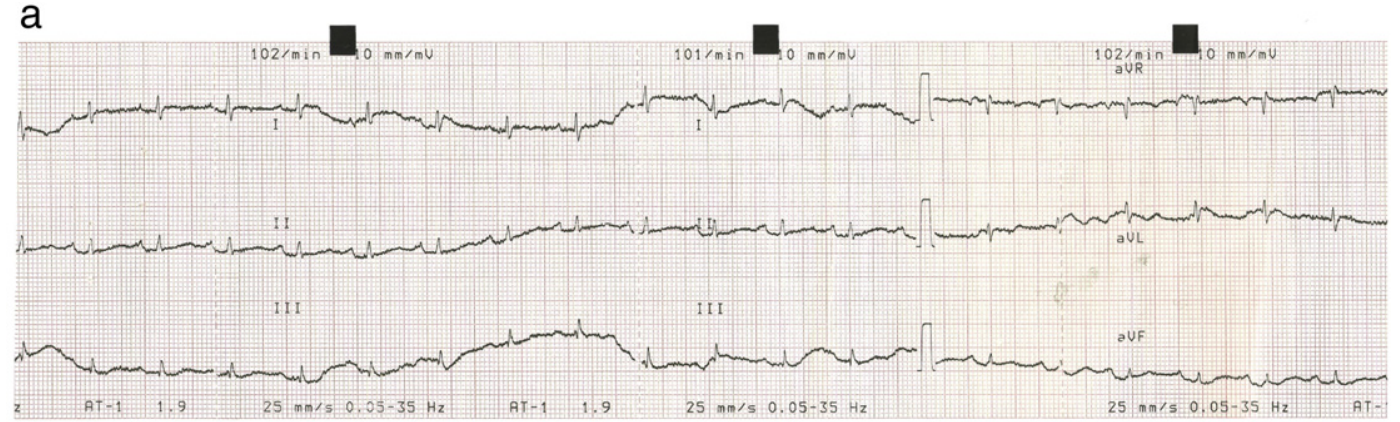

\section{b}

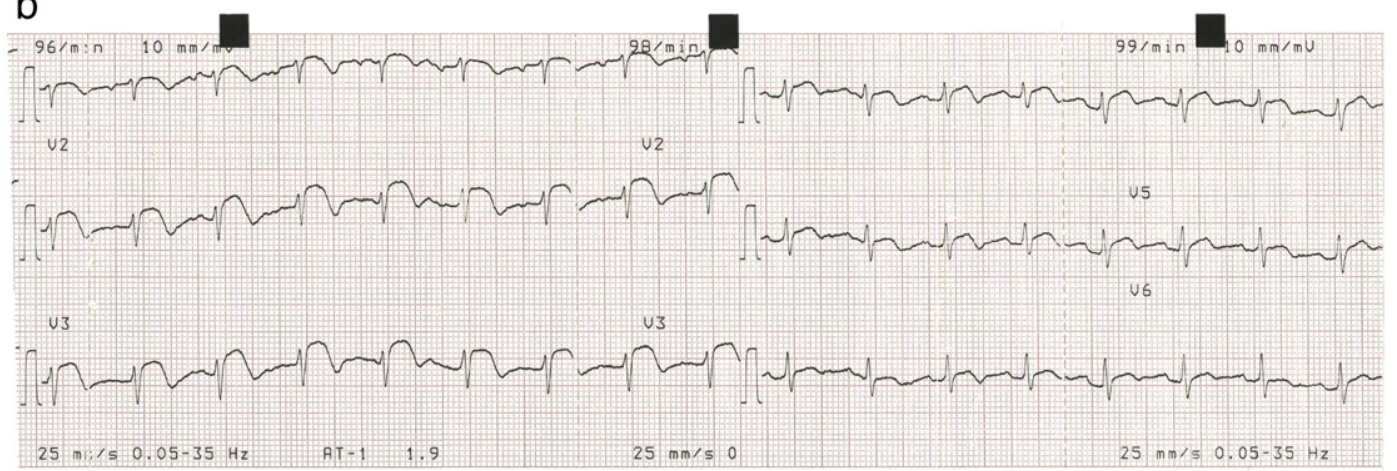

Fig. 1. Electrocardiogram showing ST elevation in V1-V5 without secondary ST depression in inferior leads.

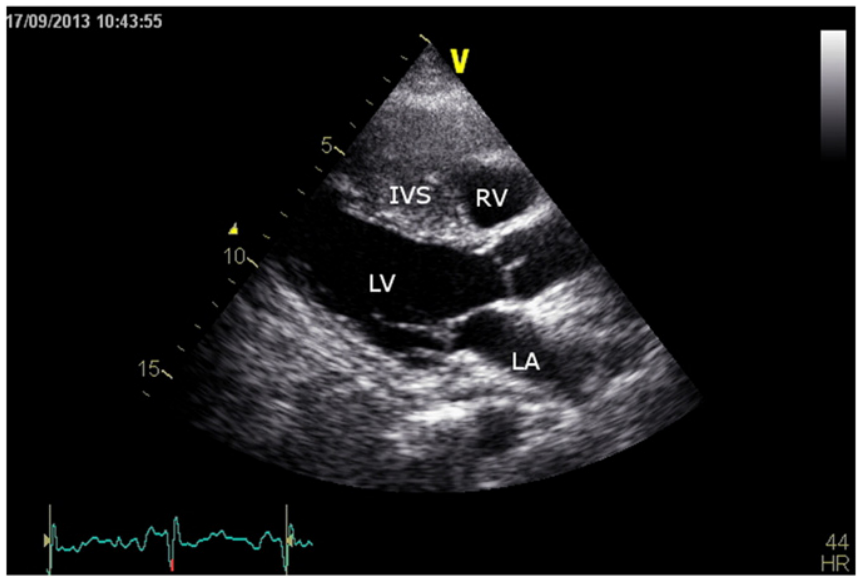

Fig. 2. Echocardiography in PLAX view. LV: left ventricle; LA: left atrium; IVS: interventricular septum; RV: right ventricle; PE: pericardial effusion.

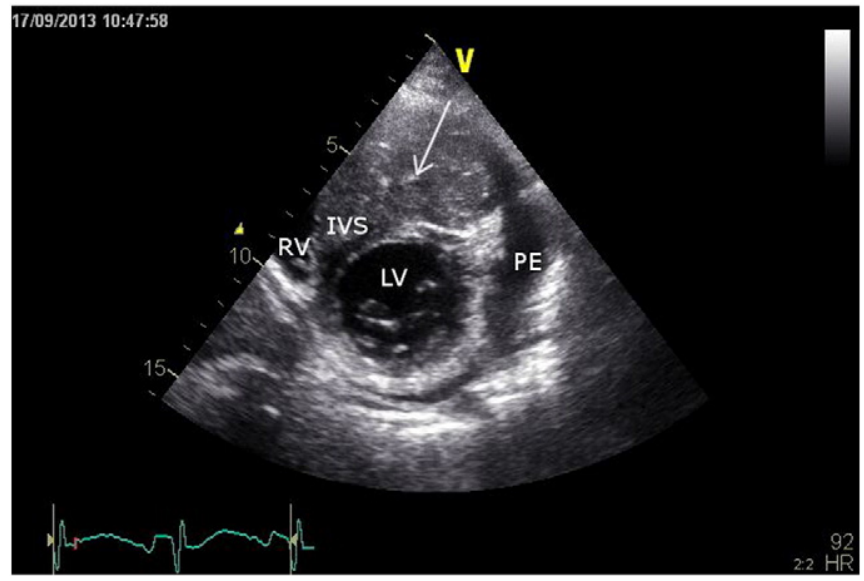

Fig. 3. Echocardiography in PSAX-MV view. LV: left ventricle; IVS: interventricular septum; RV: right ventricle; PE: pericardial effusion; Arrow: mass. 


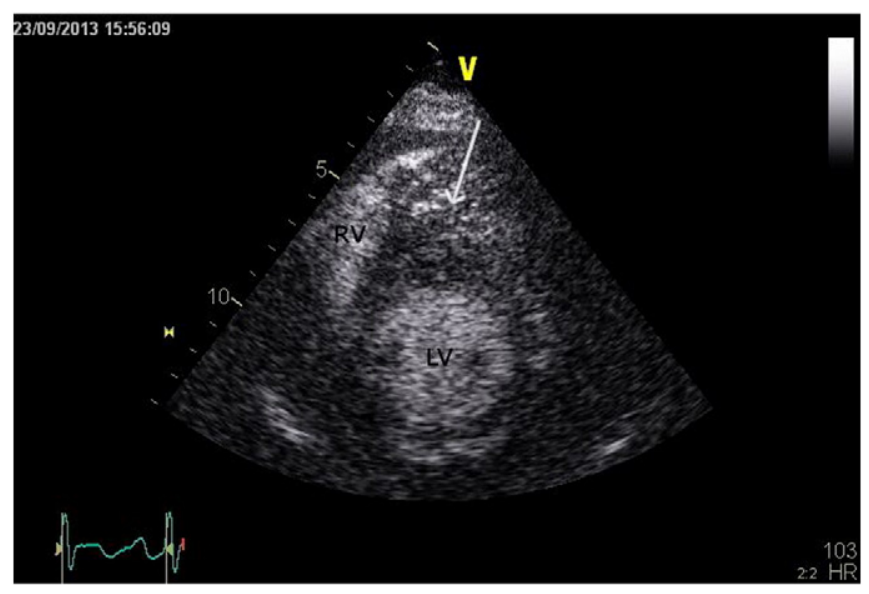

Fig. 4. Contrast echocardiography enhancing the heterogeneous aspect of the mass. LV: left ventricle; RV: right ventricle; Arrow: mass.

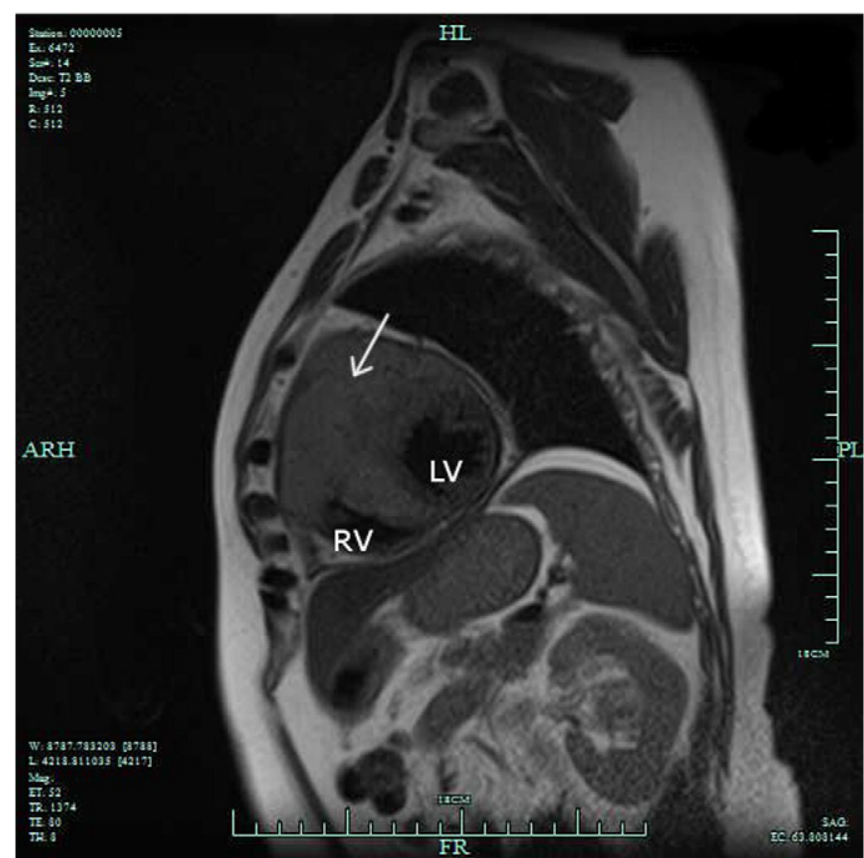

Fig. 6. Cardiac magnetic resonance. LV: left ventricle; RV: right ventricle; Arrow: mass.

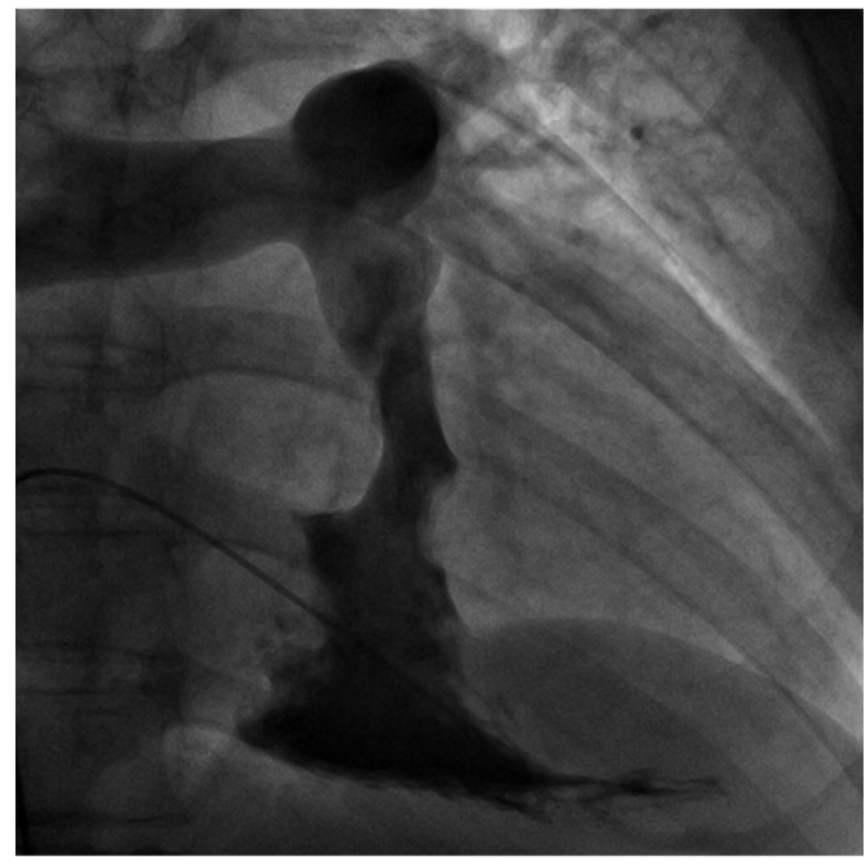

Fig. 7. Right ventriculography showing the mass occupying part of the body and outflow tract of the right ventricle. 


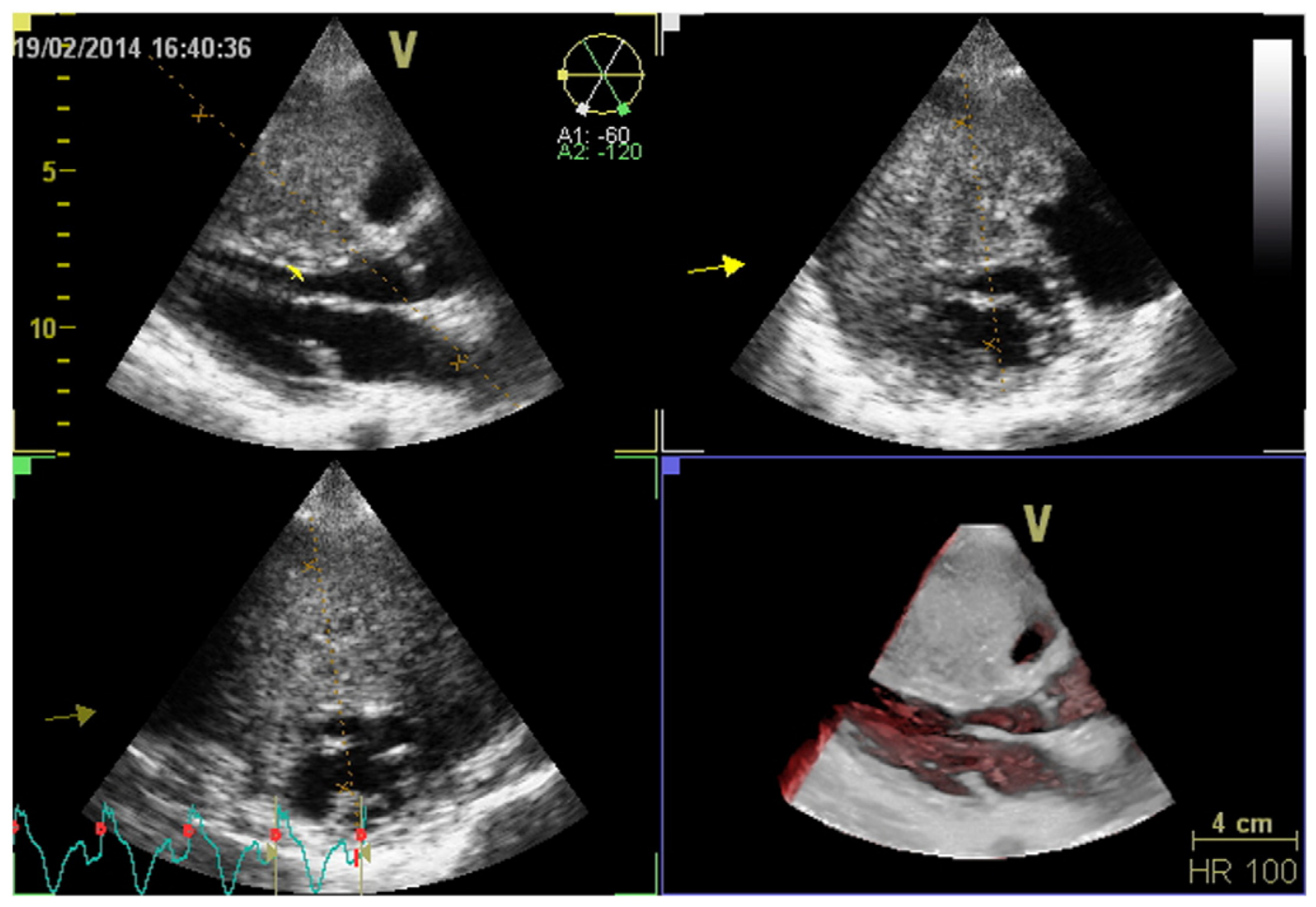

Fig. 8. 3D echocardiography showing tumor growth. 